\title{
Diseño de Objetos de aprendizaje en contextos Universitarios
}

\section{Design of Learning-object for University context}

Presentación: 28/08/2020

\section{Doctorando:}

Valeria Bertossi

Universidad Tecnológica Nacional - Facultad Regional Santa Fe - Argentina

vbertossi@frsf.utn.edu.ar

\section{Director/a:}

\section{Ma. De los Milagros Gutiérrez}

\section{Resumen}

Con el auge de la educación mediada por tecnologías (e-learning), el concepto de Objeto de aprendizaje ha tomado relevancia. Particularmente este año, donde el uso de tecnologías se volvió indispensable, dejó evidenciado las debilidades no sólo en las competencias digitales docentes sino también en las herramientas necesarias para llevar adelante las nuevas prácticas educativas mediadas por tecnologías. En este contexto, diseñar un objeto de aprendizaje que permita a los alumnos alcanzar las competencias que se pretenden, requiere no sólo de entender el concepto de objeto de aprendizaje, sino también de contar con herramientas que permitan obtener objetos de aprendizaje de calidad. En este trabajo se presentan los avances realizados sobre la conceptualización de objetos de aprendizajes, su diseño y las características de calidad que se pretenden alcanzar.

Palabras clave: objeto de aprendizaje, e-learning, calidad de objetos de aprendizaje

\begin{abstract}
With the rise of technology-mediated education (e-learning), the concept of the Learning Object has gained relevance. Particularly this year, where the use of technologies became essential, it revealed the weaknesses not only in teaching digital skills but also in the tools necessary to carry out new educational practices mediated by technologies. In this context, designing a learning object that allows students to achieve the competencies that are intended requires not only understanding the concept of learning object, but also having tools that allow obtaining quality learning objects. This work presents the progress made on the conceptualization of learning objects, their design and the quality characteristics that are intended to be achieved.
\end{abstract}

Keywords: learning object, e-learning, learning object quality

\section{Introducción}

La incorporación de tecnologías de la información y las comunicaciones (TIC) en educación (e-learning) representa un gran desafío en la renovación del modelo de enseñanza en su totalidad, que implica la búsqueda de recursos para enfrentar cuestiones complejas como la planificación, diseño e implementación de programas y cursos adecuados a la realidad (Llorente y Cabero, 2005; Cabero Almenara et al, 2004). La creciente disponibilidad del e-learning, gracias a la alta conectividad con que se cuenta 
en nuestra región, ha permitido el acceso universal con la problemática asociada de tener interlocutores muy diversos tales como profesores, alumnos, administradores, autoridades educativas, etc. (Romero et al, 2015).

En la actualidad, muchas de las actividades humanas se vieron influenciadas por el uso de las TIC. Sin embargo, en el ámbito de la educación, los cambios producidos no alcanzan para romper con los esquemas clásicos de enseñanza. Una de las necesidades es adaptar la enseñanza a las características particulares del individuo que aprende ya que no todos los estudiantes aprenden al mismo ritmo; algunos lo hacen rápido y si no se los sigue motivando pierden interés; otros aprenden más lento y si no se los estimula se sienten fracasar y abandonan (Peres et al, 2017; Sun et al, 2008). Esta situación es difícil de manejar, más aún en cursos numerosos y con alumnos dispersos físicamente. Por otro lado, los estudiantes muchas veces no saben cómo seguir si el docente no está presente físicamente, si no han aprobado ciertas evaluaciones o si no han entendido algún material asignado.

La academia, al estar inserta en la sociedad digital, exige a los docentes el aprovechamiento de las tecnologías al servicio de diversos propósitos, tanto educativos como de soporte a las tareas vinculadas a su actividad (Bustos Sánchez y Cool Salvador 2010; Cano García, 2008). Entre la vasta cantidad de herramientas de finalidad pedagógica se encuentran los objetos de aprendizaje (OA), que explotan el modo que el colectivo que ocupa las aulas de hoy aprehende la realidad con los recursos tecnológicos cotidianos (Bertossi et al, 2018). Sin embargo, diseñar un OA no es tarea trivial; requiere que el docente no sólo domine el área del saber en cuestión sino también pautas pedagógicas que permitan determinar qué OA se adecua al nivel de conocimiento pretendido y cómo, cuándo y a quién evaluar, entre otras consideraciones. En esta primera etapa se pretende entender el concepto de OA y su estructura para poder así proponer una herramienta para su diseño siguiendo pautas pedagógicas.

\section{Desarrollo}

\section{Estructura de OA.}

Si bien la definición de OA no es única, en este trabajo se adopta la siguiente: un OA es "una unidad didáctica digital independiente, cuya estructura está formada por un objetivo de aprendizaje específico, un contenido, actividades y una autoevaluación, y que puede ser reutilizada en diferentes contextos tecnológicos (Repositorios, Entornos virtuales de enseñanza y aprendizaje) y educativos. Además, cuenta con unos metadatos que propicien su localización dentro de los repositorios y permitan abordar su contextualización" (Maldonado, 2015).

En cuanto a sus propiedades, es decir las características sin las cuales un OA deja de ser tal, algunas surgen de la definición dada y otras se derivan de trabajos de otros autores, siendo la principal, sin duda, la reusabilidad (Polsani, 2003). Estas propiedades se muestran en la figura 1.

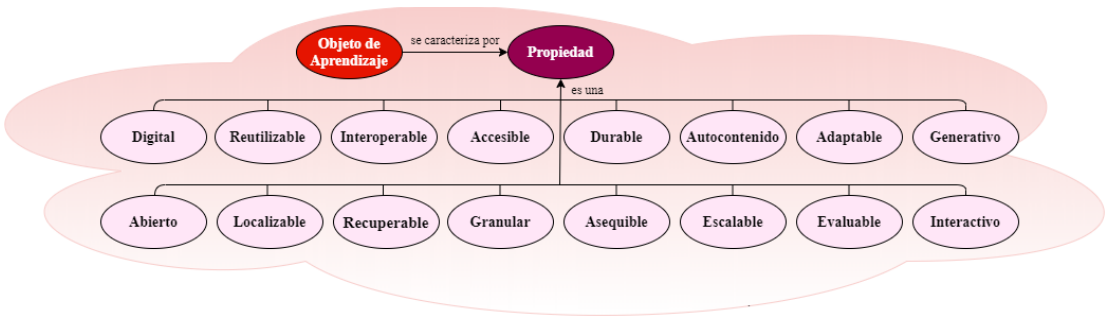

Figura 1: Propiedades de un OA.

En particular, la reusabilidad tiene una acepción tecnológica y otra pedagógica. La primera es función de la interoperabilidad, que alude a la utilización del OA dentro de distintos sistemas de gestión del aprendizaje o entornos virtuales. Para explicar la segunda, cabe hacer una distinción entre reúso y reutilización. Por reúso se entiende el uso repetido de un elemento en varias ocasiones, sin variar su función o propósito; por ejemplo, un docente da todos los años el mismo temario en su asignatura. La reutilización, en cambio, está vinculada al empleo de un mismo OA en una variedad de situaciones pedagógicas; implica cambio en las condiciones o modificación en el propósito de uso para los cuales estaba originalmente destinado (Callejas Cuervo et al, 2011; Chiappe Laverde, 2009). Así entendida, la reutilización pasa a ser función de la adaptabilidad. 
La conceptualización de un OA se aborda desde dos dimensiones, una tecnológica y otra pedagógica. La primera está vinculada a las iniciativas que se focalizaron en definir estándares y metadatos que faciliten el almacenamiento, búsqueda y recuperación de los OA en repositorios de la Web y posibiliten su interoperabilidad en plataformas de aprendizaje virtual. La figura 2 muestra el modelo conceptual de esta dimensión. Los metadatos proveen información descriptiva sobre los OA y su uso adecuado y completo, constituye uno de los criterios para evaluar la calidad de estos recursos, dado que la cantidad y riqueza de los metadatos mejora el potencial de reusabilidad.

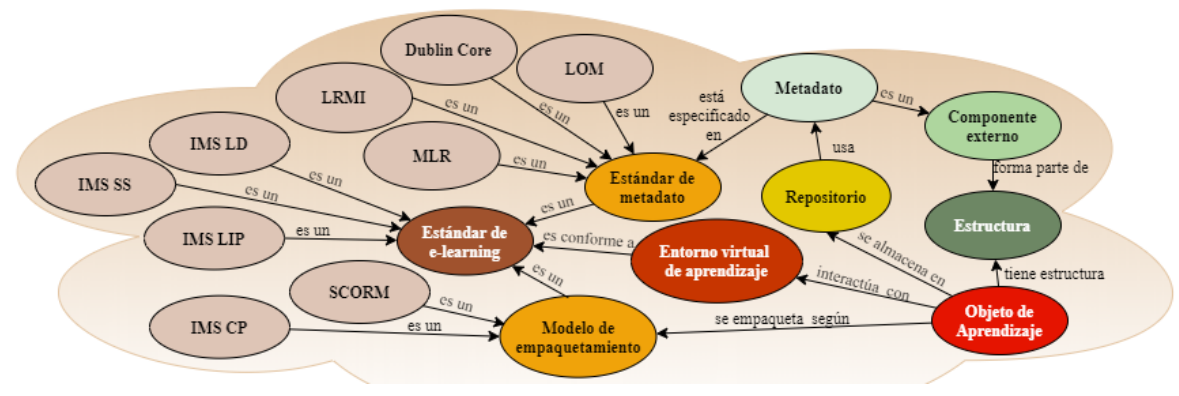

Figura 2: Dimensión tecnológica del OA.

La dimensión pedagógica, mostrada en la figura 3, es la razón de ser de los OA en tanto son concebidos como cierto material digital mediador del aprendizaje dentro del fenómeno de la incorporación de las TIC en el proceso educativo centrado en el estudiante.

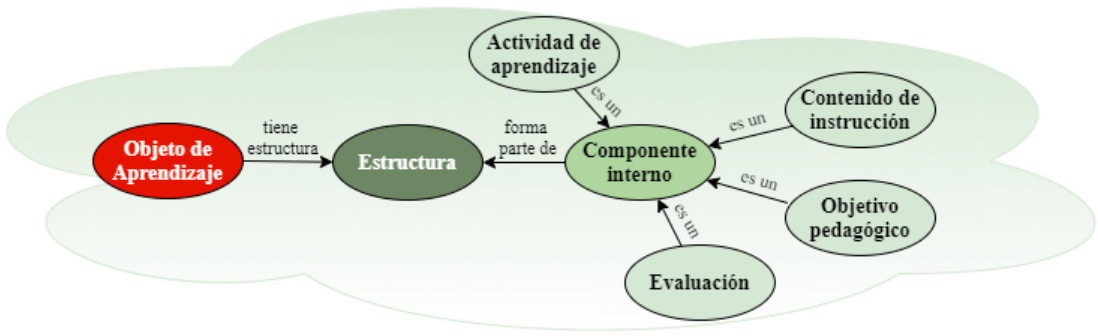

Figura 3: Dimensión pedagógica: estructura de un OA.

Cuatro componentes fundamentales se distinguen en la estructura de los OA dentro de esta dimensión (Barritt et al, 1999; Collaguazo et al, 2015; Gértrudix et al, 2007; L'Allier, 1997; Maldonado et al, 2015; MEN - Colombia, 2012; Sanz, 2015):

1) Objetivo pedagógico: es un propósito simple de aprendizaje de un contenido curricular y que encauza el aprendizaje de quien interactúa con el OA (Chiappe Laverde, 2009).

2) Contenido de instrucción: es aquello que debe ser aprendido según el currículo (Chiappe Laverde, 2009). Puede referirse a: (i) datos, conceptos, hechos y principios, (ii) procedimientos, algoritmos y procesos, o (iii) actitudes, valores, normas y reflexiones (Morales et al, 2005; Zapata Ros, 2005).

3) Actividad de aprendizaje: es la fuerza motriz del aprendizaje, el componente que genera el aprendizaje significativo y autónomo del contenido a través de la participación activa de quien aprende en la ejecución de actividades. Las mismas pueden ser de tipo memorístico o de un mayor nivel de complejidad como las que colocan al alumno en posición de analizar, reflexionar y solucionar problemas (Chiappe Laverde, 2009).

4) Evaluación: es un instrumento de autoevaluación del estudiante para determinar en qué medida ha logrado las competencias u objetivos planteados y que lo invita a reflexionar sobre lo que ha aprendido y cuánto ha aprendido (Sanz, 2015).

Estos componentes se complementan con los niveles de asimilación del aprendizaje (figura 4), los cuales corresponden a los diferentes estados cualitativos del aprendizaje (L'Allier, 1997; Butcher et al, 2011): (i) Primer nivel de asimilación: Conocer, saber que algo existe. Los alumnos retienen el conocimiento y pueden reproducirlo, pero no saben razonarlo, ni aplicarlo con 
propiedad. (ii) Segundo nivel de asimilación: Comprensión cognitiva, profundizar en el manejo de la información. Existe un verdadero aprendizaje, aunque es un saber improductivo, el alumno presenta dificultades para aplicarlo en la solución de problemas y situaciones de la vida real. (iii) Tercer nivel de asimilación: Aprendizaje profundo, manejo y aplicación de la información, comprensión autónoma. Los alumnos comprenden perfectamente los conocimientos que les han sido expuestos, saben usarlos con seguridad y aplicarlos a la solución de problemas y situaciones de la vida real.

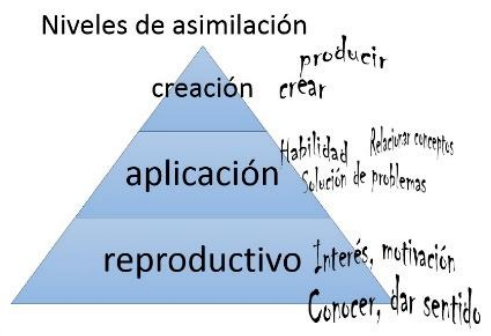

Figura 4: Niveles de asimilación.

En este sentido, cuando se diseña un OA, es importante identificar cuáles de los niveles (uno o más) se quieren lograr en el alumno. Para cada uno de estos niveles se requieren diferentes actividades de enseñanza aprendizaje que parten de clases expositivas, lecturas comentadas, videos para el primer nivel y que se van complementando con actividades más complejas como debates, ensayos, estudios de caso, de manera de alcanzar el máximo nivel. Por lo tanto, dentro de la estructura del OA, es importante identificar que tanto el componente Evaluación como Actividad de aprendizaje, tienen una estructura en sí misma, en donde los caminos de aprendizaje, aquellos que identifican el nivel en que el estudiante se encuentra, toman relevancia. En este orden de cosas, se propone el desarrollo de herramientas que den soporte al diseño de OA. A través del uso de ontologías para la conceptualización de dominios de conocimiento y de sistemas recomendadores es posible guiar al diseñador sobre aspectos a incluir en un OA.

\section{Calidad de OA}

La dimensión pedagógica de los OA circunscribe su uso, aunque no excluyente, dentro del marco educativo formal. Lo que implica que debe procurarse la calidad de los mismos previo a su incorporación en la práctica educativa para garantizar el logro de aprendizajes. Por ello es necesario que el desarrollo de OA sea una actividad interdisciplinaria, donde profesionales de la pedagogía, psicología, bibliotecología y tecnologías, además del académico que conoce el dominio de un saber particular, pongan en juego su acervo para satisfacer tres tipos de requisitos (Herrera Batista, 2002): de dominio, referente a los contenidos de la asignatura; los psicopedagógicos, que corresponden al enfoque teórico y práctico del aprendizaje de acuerdo con los paradigmas asumidos; y los de interfaz, que derivan de las características propias del medio y el nivel de interactividad que serán utilizados.

Como producto de software, los OA podrían someterse a los estándares de calidad existentes. Pero es su estatus pedagógico junto a sus propiedades inherentes lo que obliga a tener unas consideraciones particulares. Al respecto, Ruiz González et al (2007) afirman: "la calidad se refiere a características mesurables, y concretamente en el caso de los OA, por tratarse de recursos didácticos, se habla del cumplimiento de objetivos pedagógicos y del aseguramiento del aprendizaje".

Un análisis completo de los criterios de calidad en OA fue realizado en Bertossi et al (2020). En la figura 5 se pueden apreciar los aspectos de calidad agrupados según la dimensión a la que pertenecen. Los criterios de calidad de índole tecnológica involucran aspectos vinculados a la conformidad con estándares (tanto de calidad de software como de metadatos para recursos de e-learning) y al cumplimiento de las propiedades que deben reunir los OA. En tanto que los criterios de corte pedagógica cubren aspectos relacionados con el logro de los aprendizajes en el marco de un contexto educativo formal. De aquí en más los desarrollos que se continúen tendrán en cuenta los mismos. 


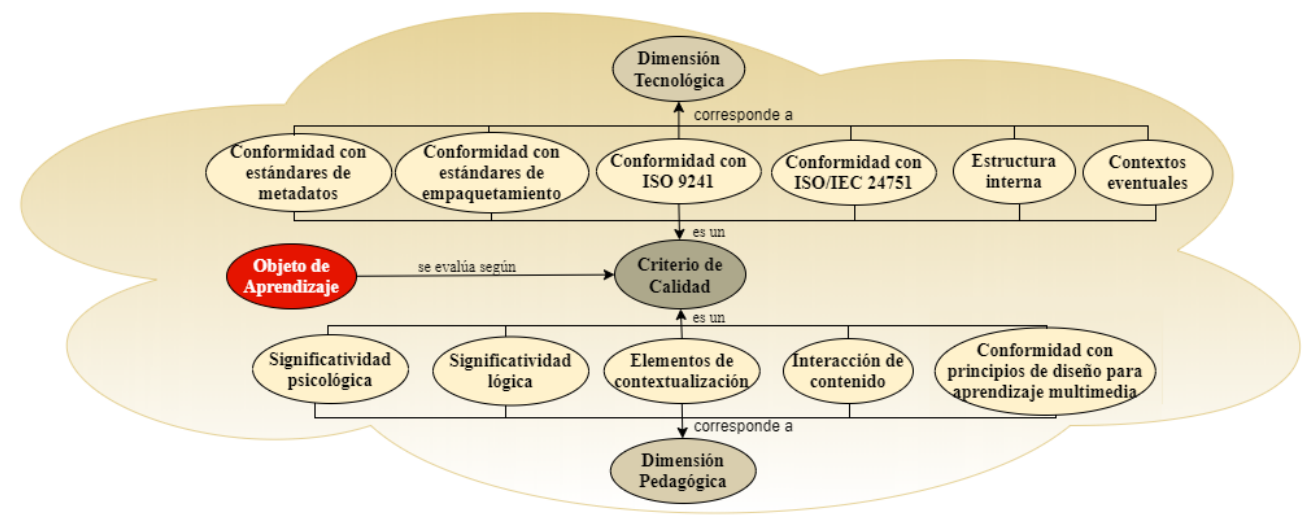

Figura 5: Criterios de calidad de OA.

\section{Conclusiones}

Los OA fueron objeto de una nutrida investigación desde principios de los '90, época en la que comenzó a popularizarse el uso de la Web para e-learning. Los distintos autores fueron utilizando diversas terminologías y definiciones para referirse a ellos. La falta de acuerdo en una conceptualización consensuada, tal vez, se deba a la multiplicidad de perspectivas desde la que los autores han abordado el asunto: educativa, tecnológica y hasta económica. Situación que obliga al investigador a adoptar una (o alguna combinación) de ellas, o bien, delinear su propia definición como marco de trabajo para llevar adelante su tarea, con argumentos fundados cualquiera sea el caso.

En la evolución del concepto OA se evidencia la convergencia de dos vertientes, una proveniente de las ciencias exactas y la otra de las ciencias sociales: la tecnológica y la pedagógica, respectivamente. La primera refiere a estándares, metadatos y repositorios en Internet que permitan la accesibilidad e interoperabilidad de los OA; mientras que la segunda hace hincapié en objetivos de aprendizaje, un contenido a aprender, actividades para aprender el contenido, evaluación que mida el objetivo e implica la necesidad de una disciplina que guíe el desarrollo de estos materiales, el Diseño Instruccional. En línea con lo aquí presentado se prevé continuar la investigación que profundice en metodologías de desarrollo de OA, demarcación del ciclo de vida de los OA, estándares de e-learning y de evaluación de calidad de OA, el estudio de repositorios, sistemas recomendadores y ensambladores de OA, e indicadores existentes de reusabilidad.

\section{Referencias}

Barritt, C., Lewis, D. y Wieseler, W. (1999). Cisco Systems Reusable Information Object Strategy: Definition, Creation Overview, and Guidelines. Cisco System, Inc.

Bertossi, V., Martinez, M., Romero, L., Gutiérrez, M. (2020). Quality Analysis of Learning Objects in Academic Contexts. LAJC Latinamerica Journal of Computing, 7(1), 100-113. Recuperado de https://lajc.epn.edu.ec/index.php/index

Bertossi, V., Pastorelli, S. y Casco, E. (2018). Objeto de Aprendizaje de Ecuaciones Diferenciales: Confluencia de Didáctica y Tecnología. I congreso iberoamericano de docentes. Algeciras. Recuperado de http://congreso.formacionib.org/1718.pdf

Bustos Sánchez, A. y Cool Salvador, C. (2010). Los entornos virtuales como espacios de enseñanza y aprendizaje. Una perspectiva psicoeducativa para su caracterización y análisis. Revista mexicana de investigación educativa, 15(44), 163-184.

Butcher, Kanwar, A. y Uvalić-Trumbić, S. (2011). A basic guide to open educational resources (OER). Vancouver, Paris: Commonwealth of Learning; UNESCO. Section for Higher Education.

Cabero Almenara, J., Llorente Cejudo, M. C. y Román Graván, P. (2004). Las herramientas de comunicación en el "aprendizaje mezclado". Pixel-Bit. Revista de Medios y Educación, (23), 27-41. Recuperado de https://idus.us.es/xmlui/bitstream/11441/22780/1/09e4150d0ad1a010d5000000.pdf

Callejas Cuervo, M., Hernández Niño, E. J. y Pinzón Villamil, J. N. (2011). Objetos de aprendizaje, un estado del arte. Entramado, 7(1), 176-189. 
Chiappe Laverde, A. (2009). Acerca de lo pedagógico en los objetos de aprendizaje. Reflexiones conceptuales hacia la construcción de su estructura teórica. Estudios Pedagógicos, 35(1), 261-272. Universidad Austral de Chile. Valdivia, Chile

Collaguazo, Y., Chamba-Eras, L. y Padilla, A. (2015). Propuesta de un modelo genérico para el diseño y valoración de Objetos de Aprendizaje basado en estándares e-learning. Anais temporários do LACLO 2015, 10(1), 227. Recuperado de https://www.br-ie.org/pub/index.php/teste/article/download/5803/4093

Gértrudix, M., Álvarez, S., Galisteo, A., Gálvez, M. d. C. y Gértrudix, F. (2007). Actions in the design and development of digital educational objects: institutional programmes. RUSC. Universities and Knowledge Society Journal, 4(1), 14-25. https://doi.org/10.7238/rusc.v4i1.296

Herrera Batista, L. M. Á. (2002). Las fuentes del aprendizaje en ambientes virtuales educativos. Reencuentro. Análisis de Problemas Universitarios, (35), 69-74.

Jaramillo Roldán, R. (2004). La calidad de la Educación: hacia un concepto de referencia. Revista Educación y Pedagogía, 16 (38), 73-90.

L'Allier, J. J. (1997). Frame of Reference: NETg's map to the products, their structure and core beliefs. NetG Whitepaper. Citado en Polsani, Pithamber R.(2003) The Use and Abuse of Reusable Learning Objects. Journal of Digital Information, 3(4).

Leyva, L. M. L., Garrido, Y. P., Leyva, J. L. L., Varona, R. C. y Rodríguez, R. R. (2007). Reflexiones sobre la evaluación de la calidad del aprendizaje en la práctica pedagógica en la escuela primaria. Revista Iberoamericana de Educación, 44(7), 3.

Llorente, M. C. y Cabero, J. (2005). Las plataformas virtuales en el ámbito de la teleformación. Revista electrónica Alternativas de Educación y Comunicación. Recuperado de https://idus.us.es/xmlui/bitstream/11441/24672/1/file_1.pdf

Maldonado, J., Sanz, C. y Fernández-Pampillón, A. (2015). Desarrollo de un Marco de Análisis para la Selección de Metodologías de Diseño de Objetos de Aprendizaje (OA) basado en criterios de calidad para contextos educativos específicos. [Tesis]. Universidad Nacional de La Plata, La Plata, Buenos Aires. Recuperado de: http://sedici.unlp.edu.ar/bitstream/handle/10915/45063/Documento_completo.pdf?sequence=3

MEN Ministerio de Educación Nacional - Colombia. (2012). Recursos Educativos Digitales Abiertos - Colombia.

Morales, E., García, F. J., Moreira, T., Rego, H. y Berlanga, A. (2005). Valoración de la calidad de unidades de aprendizaje. Revista de Educación a Distancia.

Peres, P., Oliveira, L., Jesus, Â. y Silva, A. (2017). Designing learning paths: Contributions to the organization of b-learning initiatives. 12th Iberian Conference on Information Systems and Technologies (CISTI), Lisbon, 1-6.

Polsani, P. R. (2003). Use and Abuse of Reusable Learning Objects. Journal of Digital Information, 3(4).

Romero, L. J., Ballejos, L. C., Gutiérrez, M. M. y Caliusco, M. L. (2015). Stakeholder's analysis in e-learning software process development. EAI Endorsed Transactions on e-Learning, 2(5), e4. https://doi.org/10.4108/el.2.5.e4

Ruiz González, R. E., Muñoz Arteaga, J. y Álvarez Rodríguez, F. J. (2007). Evaluación de Objetos de Aprendizaje a través del Aseguramiento de Competencias Educativas. VIII Encuentro Internacional Virtual Educa Brasil 2007.

Sanz, C. V. (2015). Los objetos de aprendizaje, un debate abierto y necesario. Bit \& Byte, 1(1). Recuperado de http://sedici.unlp.edu.ar/bitstream/handle/10915/46722/Documento_completo__.pdf?sequence=1

Sun, P.-C., Tsai, R. J., Finger, G., Chen, Y.-Y. y Yeh, D. (2008). What drives a successful e-Learning? An empirical investigation of the critical factors influencing learner satisfaction. Computers \& Education, 50(4), 1183-1202. https://doi.org/10.1016/j.compedu.2006.11.007

Zapata Ros, M. (2005). Secuenciación de contenidos y objetos de aprendizaje. RED: Revista de Educación a Distancia, 1. 